gene product inhibits quiescent cells from entering $\mathrm{S}$ phase after serum stimulation in certain cell types. However, the mRNAs described by Bresnahan and Shenk ${ }^{3}$ are likely to encode proteins that are required for the viral function at early stages of infection, before the viral genome becomes transcriptionally active.

Virally encoded glycoproteins located in the virion envelope also induce considerable cellular changes when they bind their host cell receptors. Cellular transcription factors, such as NF- $\mathrm{KB}$ and Sp1, are activated upon viral absorption (Fig. 1). This, in turn, activates downstream viral gene expression as well as cellular macromolecule synthesis ${ }^{9}$. Furthermore, binding of HCMV to human monocytes induces immunoregulatory gene expression and upregulates cell cytokine synthesis ${ }^{10}$, indicating that viral absorption can alter cell metabolism even if the infection is nonproductive. Thus, the infectious virion does not merely introduce viral DNA to the nucleus of infected cells, but also induces many cellular changes that may create an environment favorable for viral replication. These virion-mediated changes may induce the host cell to enter an active metabolic state that leads to cell proliferation if the infection is nonproductive

The Bresnahan and Shenk paper raises many interesting questions. Are these mRNAs specifically packaged and, if so, how? It is possible that they form complexes with other tegument proteins to localize within the virion. Alternatively, as these mRNAs are present at very high levels during virion assembly, they may be taken up into the tegument non-specifically. It will al so be of interest to determine the function of these mRNAs. The mRNAs may have a non-protein-coding function, playing a structural role in the assembly and organization of the tegument proteins. Alternatively, mRNAs or their products may facilitate the expression of the viral DNA genome or the transportation of capsid-tegument structures to nuclear pore ${ }^{2,3}$. These questions could be answered by analysis of mutant viruses that do not possess these mRNAs.

Different types of herpesviruses share many structural, biological and biochemical properties. Their genes share strong homology and are even colinear, and all herpes virions contain a tegument layer. Other herpesviruses may also package similar types of mRNAs within their virions. Determining which viruses contain both DNA and RNA will affect classification of the herpesvirus family. Discovering the function that the virion mRNAs themsel ves or their protein products play in HCMV pathogenesis will be of great interest to virologists and may lead to new targets for treatment of herpesvirus infections.

1. Mocarski, E.S. in Fields Virology, 3rd edn. Vol. 2 (ed. Fields, B.N.) 2447-2492 (Lippincott-Reven, Philadelphia, 1996)

2 Roizman, B. Redefining virology. Science 288, 2327-2328 (2000).

3. Bresnahan, W.A. \& Shenk, T. A subset of viral transcripts packaged within human cytomegalovirus particles. Science 288, 2373-2376 (2000).

4. Mar, E.-C. et al. Human cytomegalovirus-associated DNA polymerase and protein kinase activities. J. Gen. Virol. 57,149-156 (1981).

5. Benson, J.D. \& Huang, E.-S. Human cytomegalovirus induces expression of cellular topoisomerase II. J. Virol. 64, 9-15 (1990).

6. Michelson, S. et al. Human cytomegalovirus carries serine/threonine protein phosphatases PP1 and a host-cell derived pp2A. J. Virol. 70, 1415-1423 (1996).

7. Liu, B. \& Stinski, M.F. Human cytomegalovirus contains a tegument protein that enhances transcription from promoters with upstream ATF and AP-1 cis-acting elements. J. Virol. 66, 4434-4444 (1992).

8. Hayashi, M.L. et al. Human cytomegalovirus UL69 protein is required for efficient accumulation of infected cells in the G1 phase of the cell cycle. Proc. Natl. Acad. Sci. USA 97, 2692-2696 (2000).

9. Yurochuko, A.D. et al. The human cytomegalovirus UL55 (gB) and UL75 (gH) glycoprotein ligands initiate the rapid activation of Spl and NF-kB during infection. J. Virol. 71, 5051-5059 (1997).

10. Yurochko, A.D. \& Huang, E.-S. Human cytomegalovirus binding to human monocyte induces immunoregulatory gene expression. J. Immunol. 162, 4806-4816 (1999).

Department of Medicine and Lineberger

Comprehensive Cancer Center

University of North Carolina at Chapel Hill

Chapel Hill, North Carolina 27599

Email: eshuang@med.unc.edu

\title{
Lost in the FOG
}

Targeted mutations affecting virtually all aspects of heart development have been described and provide insight into the molecular basis of congenital disorders such as ventricular septal defects, overriding aorta and myocardial defects. In the 23 June issue of Cell, Tevosian et al. describe the unique phenotype of defectivecoronary vascular development in mice lacking FOG-2. FOG-2 is a transcription factor expressed predominantly in cardiac and nervous system tissues. It physically interacts with another group of transcription factors known to be involved in development, GATA-4/5/6. Tevosian et al. observed that disruption of FOG-2 in mice caused embry- onic lethality due to ventricular thinning, a common atrioventricular canal, and heart malformations similar to those observed in to the subepicardial coronary vasculature. Thistransition dependson growth factorsignaling between the myocardium and epi-
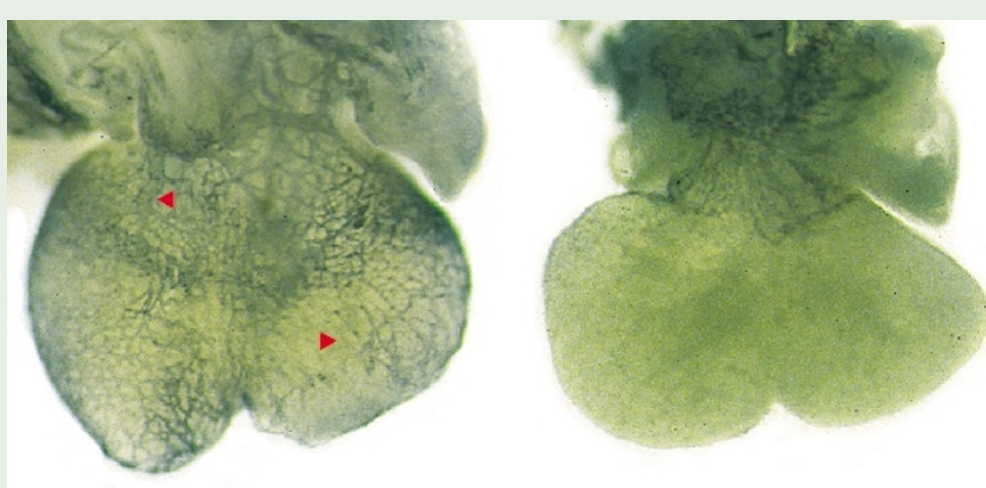
cardium, and FOG-2 may be involved in this signaling cascade. The absence of a vascular network in the ventricular myocardium can beseen through the diminished expression of the vascular marker ICAM-2. The image shows ICAM-2 staining of wild-type embryonic hearts (left) and $\mathrm{FOG}_{-2^{-1-}}$ hearts (right). The authors sug-

the tetralogy of Fallot. The most striking finding, an early block in formation of coronary vessels, is believed to be dueto the failure of FO G-2 ${ }^{-1-}$ mice to undergo the epithelial-tomesenchymal transition required to give rise gest that FOG-2 or GATA factors should be evaluated as candidate genes for congenital defects in humans.

KrIStine NovaK 